\title{
LexEQUAL: Supporting Multilexical Queries in SQL
}

\author{
A. KUMARAN *
}

\section{Introduction}

Current database systems offer support for storing multilingual data [2], but are not capable of querying across languages, an important consideration in today's global economy. We therefore propose a new multilexical operator called LexEQUAL, that extends the standard lexicographic matching in database systems to matching of text data across languages, specifically for names, which form close to twenty percent of text corpora.

Our implementation of the LexEQUAL operator is based on transforming matches in language space into parametrized approximate matches in the equivalent phoneme space. A detailed evaluation of our approach on a real data set shows that there exist settings of the algorithm parameters with which it is possible to achieve both good recall and precision.

\section{The LexEQUAL Operator}

\begin{tabular}{|c|c|c|c|c|}
\hline Author & Author_FN & Title & Price & Language \\
\hline Descartes & René & Les Méditations Metaphysiques & $€ 49.00$ & French \\
\hline G.5(5) & ஜஹஹர்ará & ஆभிய ஜோक & INR 250 & Tami 7 \\
\hline |بهنسي ، د & عفيف & التعمارة عبر التاريخ & SAR 75 & Arabic \\
\hline Nehru & Jawaharlal & Discovery of India & $\$ 9.95$ & English \\
\hline Durant & wil1 & History of Civilization & $\$ 149.75$ & English \\
\hline नेहर & जबाहरलाल & भारत एक खोज & INR 175 & Hindi \\
\hline
\end{tabular}

Catalog of Multilingual Books.com

Consider a hypothetical Books.com that sells books in different languages, with a sample product cata$\log$ as shown above, and a user query to retrieve all the works of an author, in a set of specified languages. This can be easily achieved using LexEQUAL as shown below: SELECT * FROM BOOks WHERE Author LexEQUAL 'Nehru' Threshold 0.3 IN \{ English, Hindi, Tamil, Arabic \}

The output for this query will contain all records of the above Books.com table, for whom the Author attribute contains names that are phonemically close to "Nehru", with the Threshold parameter in the query determining the match quality tradeoff between precision and recall.

* Department of Computer Science and Automation, Indian Institute of Science, Bangalore, INDIA. \{kumaran, haritsa\}@csa.iisc.ernet.in

\author{
JAYANT R. HARITSA *
}

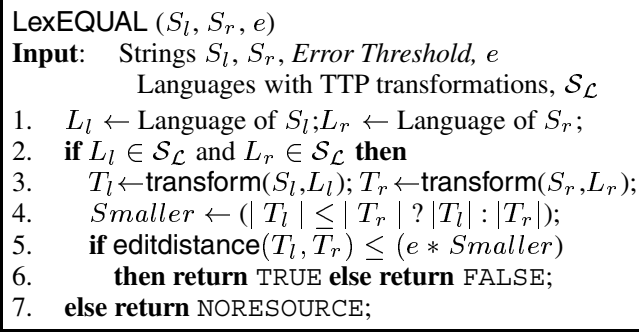

The pseudocode of our implementation of LexEQUAL is given above. The algorithm transforms the input multilingual strings to their equivalent phoneme strings and flags a match if the edit distance between them is less than the userspecified error limit, Threshold. The transform function uses standard Text-to-Phoneme (TTP) converters that convert to a canonical International Phonetic Association's phonemic alphabet. Further, the LexEQUAL implementation is parameterized for different cost functions of editdistance function and for domain-specific clustering of similar phonemes.

We have currently implemented LexEQUAL as a user-defined function, incorporating $Q$-Grams [1] to filter out non-matches inexpensively and Phonemic Indexes [4] to narrow the search to potential matches using standard database index structures. Our initial results on a commercial database system hold out the promise that an inside-the-server implementation of multilingual matching will have runtimes comparable to traditional monolingual matching.

In summary, the LexEQUAL operator employing phonetic matching can complement the standard lexicographic operators, representing a first step towards achieving complete multilingual functionality in database systems. The full version of this paper is available in [3].

Acknowledgements This work was supported in part by a Swarnajayanti Fellowship from the Dept. of Science and Technology, Govt. of India.

\section{References}

[1] L. Gravano et al. Approximate String Joins in a Database (almost) for Free. Proc. of 27th VLDB Conf., 2001.

[2] A. Kumaran and J. Haritsa. On the Costs of Multilingualism in Database Systems. Proc. of 29th VLDB Conf., 2003.

[3] A. Kumaran and J. Haritsa. Supporting Multiscript Matching in Database Systems. Proc. of 9th EDBT Conf., 2004.

[4] J. Zobel et al. Phonetic String Matching: Lessons from Information Retrieval. Proc. of 19th ACM SIGIR Conf., 1996. 